\title{
BJSM
}

\section{Descriptive profile of scapulothoracic position, strength and flexibility variables in adolescent elite tennis players}

Ann M Cools, Fredrik R Johansson, Dirk C Cambier, et al.

Br J Sports Med 2010 44: 678-684

doi: 10.1136/bjsm.2009.070128

Updated information and services can be found at:

http://bjsm.bmj.com/content/44/9/678.full.html

\section{These include:}

References This article cites 41 articles, 15 of which can be accessed free at: http://bjsm.bmj.com/content/44/9/678.full.html\#ref-list-1

Article cited in:

http://bjsm.bmj.com/content/44/9/678.full.html\#related-urls

Email alerting Receive free email alerts when new articles cite this article. Sign up in the service box at the top right corner of the online article.

\section{Notes}

To order reprints of this article go to:

http://bjsm.bmj.com/cgi/reprintform

To subscribe to British Journal of Sports Medicine go to: 


\title{
Descriptive profile of scapulothoracic position, strength and flexibility variables in adolescent elite tennis players
}

\author{
Ann M Cools, ${ }^{1}$ Fredrik R Johansson, 2,3 Dirk C Cambier, ${ }^{1}$ Annemie Vande Velde, \\ Tanneke Palmans, ${ }^{1}$ Erik E Witvrouw ${ }^{1}$
}

'Department of Rehabilitation Sciences and Physiotherapy, University Hospital, Ghent, Belgium

${ }^{2}$ Sportmedicin/ESTESS official clinic, Långängskroken 3 , Segeltorp, Sweden ${ }^{3}$ Swedish Tennis Federation, Lidingövägen 75, Stockholm, Sweden

\section{Correspondence to} Professor Ann M Cools, Department of Rehabilitation Sciences and Physiotherapy, University Hospital Ghent, De Pintelaan 185, UZ REVAKI 2B3, Gent B9000, Belgium; ann.cools@ugent.be

Accepted 31 December 2009

\author{
ABSTRACT \\ Study design Descriptive study, cross-sectional \\ design. \\ Background Tennis requires repetitive overhead \\ movement patterns that can lead to upper extremity \\ injury. The scapula plays a vital role in injury-free playing. \\ Scapular dysfunction has been associated with shoulder \\ injury in the overhead athlete.
}

Objectives The purpose of this study was to describe variables regarding scapular position, muscle strength and flexibility in young elite tennis players.

Methods Thirty-five adolescent Swedish elite tennis players (19 boys, aged $13.6( \pm 1.4)$ years, 16 girls, aged $12.6( \pm 1.3)$ years), selected on the basis of their national ranking, underwent a clinical screening protocol consisting of: scapular upward rotation at several angles of arm elevation; isometric scapular muscle strength; and anthropometric measurement of pectoralis minor (PM) length.

Results The players showed significantly more scapular upward rotation on their dominant side $(p<0.001)$. For both genders, upper trapezius $(p=0.003)$ and serratus anterior $(p=0.01)$ strength was significantly greater on the dominant side, whereas middle and lower trapezius strength showed no side differences. PM was shorter on the dominant side $(p<0.001)$, and in the female players $(p=0.006)$ compared with the boys.

Conclusion These results indicate some sportsrelated adaptations of young tennis players on their dominant side at the scapulothoracic level to exposure to their sport. These data may assist the clinician in the prevention and rehabilitation of sport-specific injuries in adolescent tennis players.

The shoulder is at great risk of injury in tennis because it faces high loads and forces during serving and hitting. Injury risk seems to increase with age, ${ }^{12}$ and despite some lack of evidence has been suggested to be related to the level and volume of play. ${ }^{2}$ Most of the reported shoulder injuries are strains, implicating a process over time, with chronic overload leading to injury. ${ }^{1}$ Chronic shoulder pain in the overhead athlete is often attributed to sport-specific adaptations, alterations in strength, flexibility and posture, not only in the glenohumeral joint, but also in other links of the kinetic chain. ${ }^{4-8}$ These alterations change biomechanics and movement strategies during serving and hitting, possibly leading to overload injuries at the shoulder. In particular, loss of glenohumeral range of motion, rotator cuff strength imbalance, scapular dyskinesis, thoracic spine stiffness, lumbar core instability and hip range of motion and strength deficits possibly create a kinetic chain 'breakage' and a 'cascade to injury', as defined by Kibler", Kibler and McMullen ${ }^{10}$ and Lintner et al. ${ }^{8}$

Numerous studies have reported the specific rotational adaptations of the glenohumeral joint ${ }^{11-15}$ and muscle balance alterations in the rotator cuff. ${ }^{16-18}$ However, studies regarding scapular dyskinesis ${ }^{1920}$ in tennis players are scarce. In a recent study, Silva et al ${ }^{20}$ described scapular dyskinesis in $43.4 \%$ of elite tennis players between 11 and 17 years of age. In addition, the study demonstrated a significantly smaller subacromial space in dyskinesis-affected athletes, possibly leading to sport-specific impingement symptoms. ${ }^{6} 20$ However, the assessment of the players in that study was based on clinical observation, rather than standardised numerical data. Moreover, exploration of these numerical variables in a population of young (10-16 years) elite players has not yet been performed. Therefore, the purpose of this study was to generate a descriptive profile of scapulothoracic position and strength variables in elite junior tennis players. In particular, scapular rotational position, trapezius and serratus muscle strength, and pectoralis minor (PM) muscle length were of interest.

\section{METHODS}

\section{Subjects}

Thirty-five adolescent Swedish elite tennis players, selected by the Swedish Tennis Federation onthe basis of their national ranking, were tested in this investigation. Nineteen boys (mean age $13.6 \pm 1.4$ years, mean height $163.6 \pm 12 \mathrm{~cm}$, mean weight $53 \pm 11.8 \mathrm{~kg}$ ) and 16 girls (mean age $12.6 \pm 1.3$ years, mean height $156.6 \pm 10.5 \mathrm{~cm}$, mean weight $46.1 \pm 7.8 \mathrm{~kg}$ ) participated in the study. All but one player were right-handed. All players used a two-handed backhand stroke. Players had been taking part in competition for approximately 7.1 $( \pm 1.4)$ years, and reported playing $13.9 \pm 2.4 \mathrm{~h}$ tennis/week. All subjects were free from any upper extremity injury at the time of testing as well as 6 months before data collection. The study was approved by the Ethical Committee of the Ghent University, and performed in collaboration with the Swedish Tennis Federation. The players were tested during a high performance physical training camp. 


\section{Measurement procedure}

Scapular inclination

Scapular upward rotation was measured using the Pro 3600 Digital Inclinometer (SPI-Tronic; Penn Tool Co, Maplewood, New Jersey, USA), using the methods originally described by Johnson et al, ${ }^{21}$ and previously used by other researchers. ${ }^{22-25}$ Two Y-shaped adjustable plastic locator rods, designed to rest comfortably over the bony contours of the spine of the scapula were attached to the inclinometer. ${ }^{2123} \mathrm{~A}$ bubble level was attached to ensure minimal anterior-posterior tilting of the inclinometer around an axis parallel to the scapular spine.

Scapular upward rotation was measured in three positions: $0^{\circ}, 90^{\circ}$ and $180^{\circ}$ of elevation in the scapular plane $\left(30^{\circ}\right.$ angle from the frontal plane as determined by goniometric measurement; figure 1). All procedures were repeated two times. This method showed good to excellent intraexaminer reliability ${ }^{21} 23$ and good to excellent criterion validity. ${ }^{21}$

\section{Scapular muscle strength}

The isometric muscle strength of the scapular muscles was measured using a hand-held dynamometer (HHD; compuFET; Hoggan Health Industries Inc, West Jordan, Utah, USA). The reliability and validity of $\mathrm{HHD}$ in the assessment of upper extremity muscle strength has been documented and found to be acceptable for clinical and research use. ${ }^{26-28}$

The upper trapezius (UT) muscle test was performed as described by Hislop et al. ${ }^{29}$ The HHD was placed over the superior aspect of the scapula. Force was applied directly downward (inferior) in the direction of scapular depression. The subject was asked to perform scapular elevation against resistance of the dynamometer (figure 2).

The middle trapezius (MT) and lower trapezius (LT) were tested in the prone position, according to Donatelli et al. ${ }^{30}$ The MT was tested with the shoulder abducted to $90^{\circ}$ with full glenohumeral external rotation. Pressure was applied at the wrist of the subject, at the lateral aspect of the distal radius. The subject was asked to perform a horizontal abduction with external rotation (figure 3).

For the LT strength measurement, the shoulder was placed into $145^{\circ}$ of abduction with full external rotation. Subjects were asked to lift up their arm, and the examiner applied

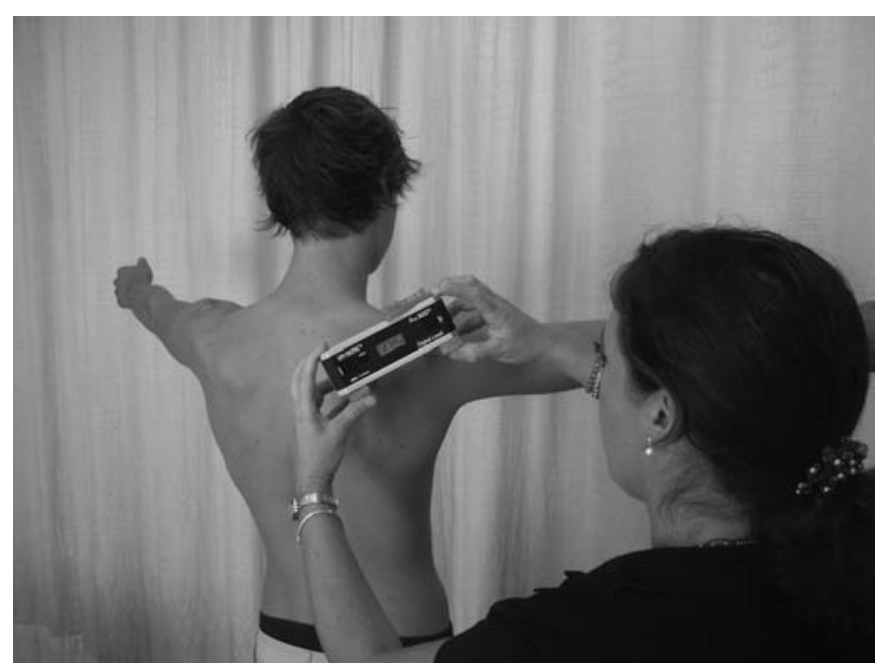

Figure 1 Scapular upward rotation measurement in $90^{\circ}$ of scapular elevation. Written consent from the patient and his legal guardian was obtained for publication of the image in print and online.

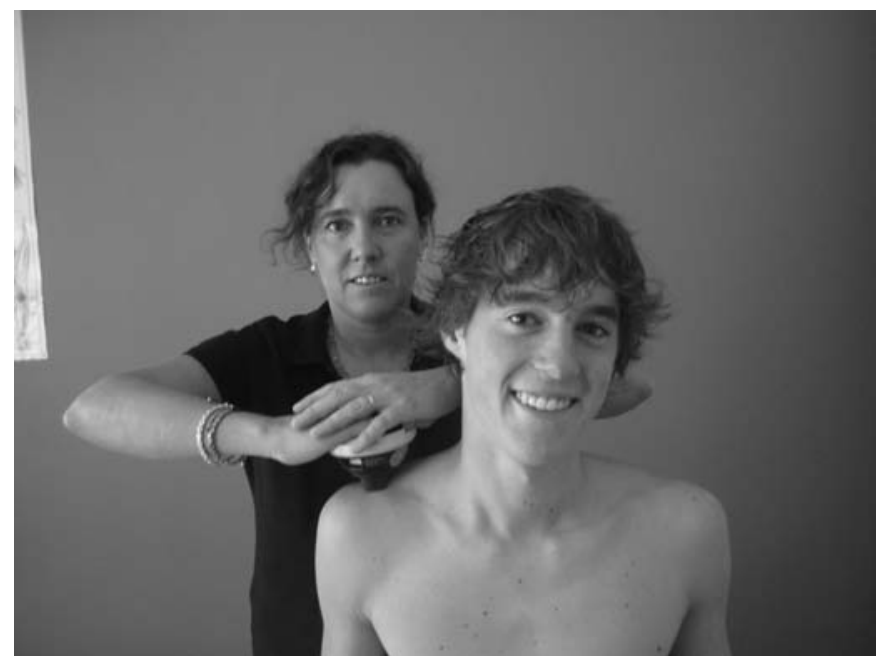

Figure 2 Manual muscle testing of the upper trapezius. Written consent from the patient and his legal guardian was obtained for publication of the image in print and online.

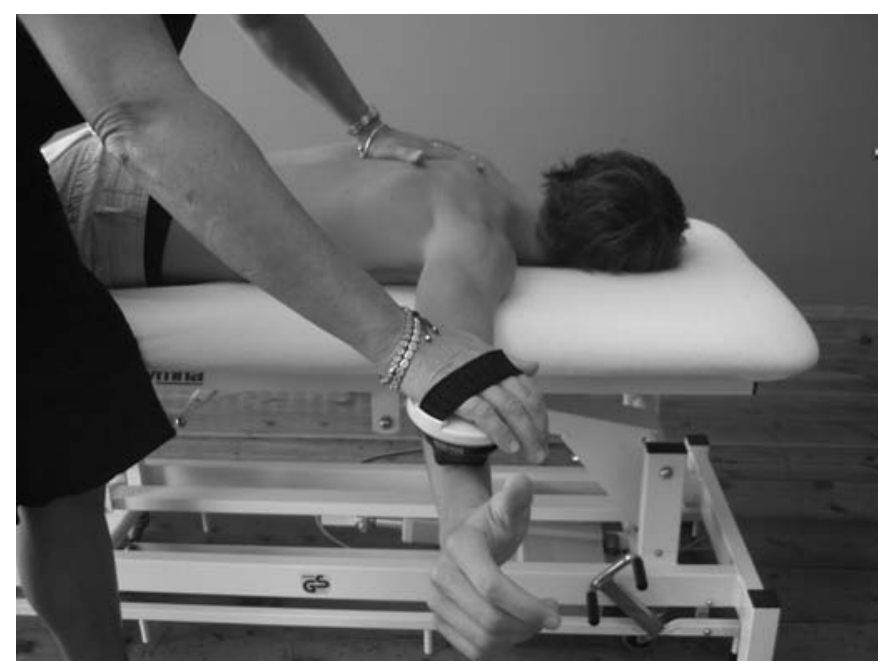

Figure 3 Manual muscle testing of the middle trapezius. Written consent from the patient and his legal guardian was obtained for publication of the image in print and online.

force at the lateral aspect of the radius in downward direction (figure 4).

For serratus anterior (SA) strength measurement, Kendall and Kendall recommendations were used. ${ }^{31}$ Subjects were supine, with the arm in $90^{\circ}$ of forward flexion. The examiner put the HHD in the palm of the hand of the subject, applying force towards the bench. The subject was asked to perform a protraction movement with the elbow extended (figure 5).

All subjects performed the manual muscle test in the same order: UT, MT, LT, SA. The non-dominant arm was tested first. Each muscle test was repeated twice, ${ }^{32}$ using a 'make test' for strength measurement. ${ }^{28}$ Data were collected and directly stored by the software program compuFET Biometrics version $1.0 \mathrm{~A}$. In addition to the absolute and weight-normalised strength data (figure 6), generated by the software program, muscle balance ratios were calculated (UT/MT, UT/LT, UT/SA and LT/SA) by dividing the muscle strength of one muscle by the other muscle. 


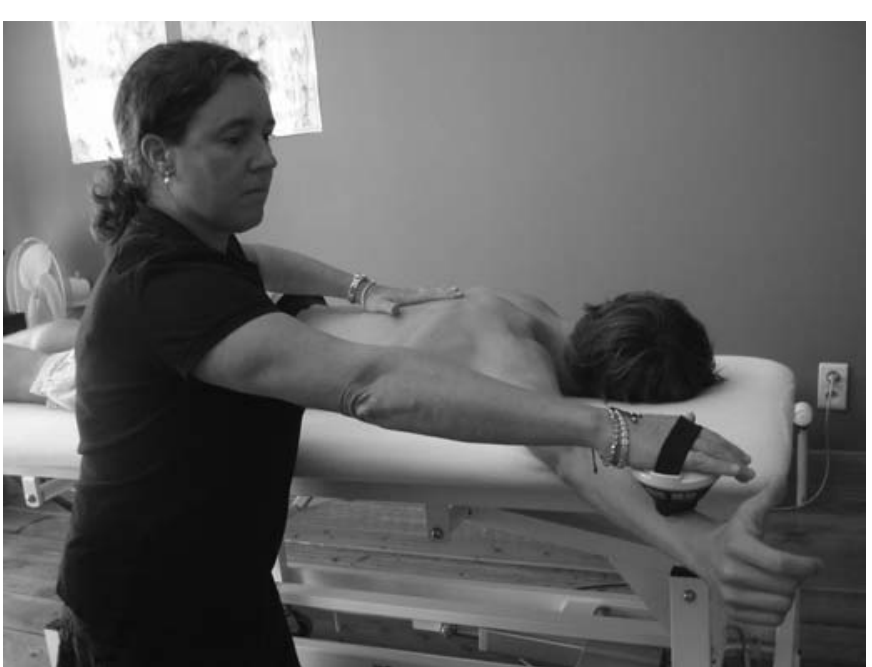

Figure 4 Manual muscle testing of the lower trapezius. Written consent from the patient and his legal guardian was obtained for publication of the image in print and online.

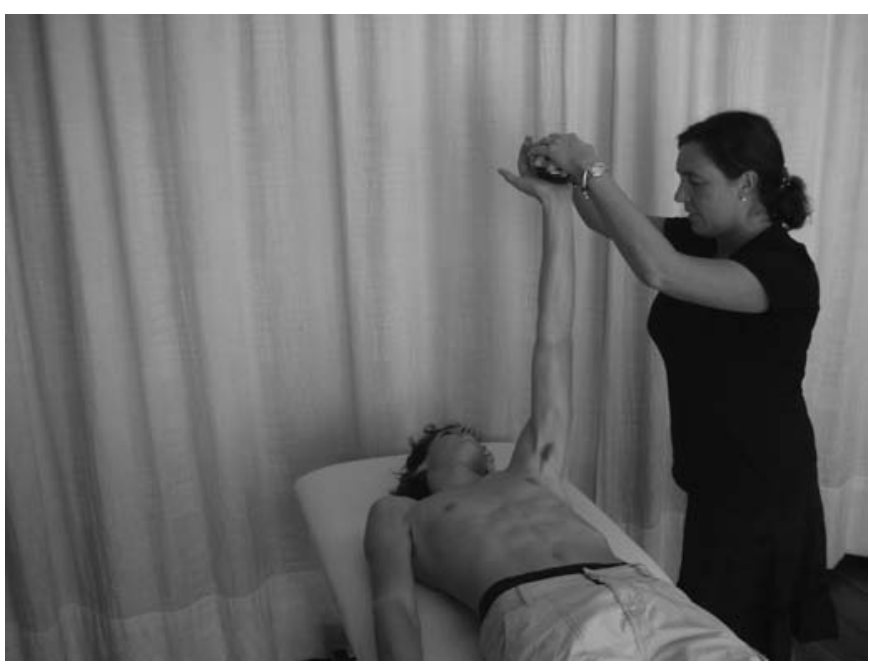

Figure 5 Manual muscle testing of the serratus anterior. Written consent from the patient and his legal guardian was obtained for publication of the image in print and online.

\section{PM length}

The anthropometric length measurement of the PM was performed according to the protocol, described by Borstad, ${ }^{33}$ slightly adapted for the purpose of this study. Before length measurement, two anatomical landmarks were determined, representing the length of the PM: the medial-inferior angle of the coracoid process and a second landmark just lateral to the sternocostal junction of the inferior aspect of the fourth rib. A Vernier calliper was used to measure the distance between both bony reference points (figure 7). The investigator was blinded from reading the result until after the measurement. Each side was measured twice; the non-dominant side was always measured first. In contrast to the protocol of Borstad and Ludewig, ${ }^{34}$ who performed the measurements in the standing position, we examined the pectoralis minor muscle length (PML) in the supine position with the elbows extended, in order to avoid postural influences of the thoracic spine and optimise muscle relaxation in all surrounding muscles. Because of height and muscle length variability, a normalisation index was used. ${ }^{34}$ The pectoralis minor index (PMI) was calculated by dividing the resting muscle length by the subject's height and multiplying by 100 .

\section{Statistical analysis}

Descriptive statistics were calculated for all variables, and all dependent variables were controlled for normal distribution and homogeneity of variance using the one-sample Kolmogorov-Smirnov test and the Levene's test. As all data were normally distributed with equal variances, parametric statistics were performed.

In order to select dependent variables for further analysis, we used independent $t$ tests to analyse group differences with respect to the anthropometric characteristics of the players. The results showed significant age differences $(t=2.211$, $p=0.034)$, but no significant differences in height $(t=1.785$, $p=0.084)$ and weight $(t=2.01, p=0.053)$. In view of the fact that group differences are not highly conclusive, being in the proximity of the $\alpha$ level of 0.05 , we decided to present absolute as well as relative results (normalised to body weight or body height), when necessary and appropriate for the goal of the study.

The dependent variables of interest were: scapular upward rotation in $0^{\circ}, 90^{\circ}$ and $180^{\circ}$ of elevation in the scapular plane (in degrees); absolute $(\mathrm{N})$ and weight-normalised $(\mathrm{N} / \mathrm{kg})$ isometric muscle strength of the UT, MT, LT and SA, and muscle balance ratios and absolute (in $\mathrm{cm}$ ) and normalised PML.

Differences in scapular upward rotation were analysed using a general linear model three-way analysis of variance (ANOVA) for repeated measures, in which the within-subject factors were side (two levels) and position (three levels), and the between-subject factor was gender (two levels).

Differences in muscle strength, muscle balance ratios and PML were analysed with a general linear model two-way ANOVA for repeated measures, in which the within-subject factor was side (two levels), and the between-subject factor was gender (two levels).

In the ANOVA analysis, three-way interactions (side $\times$ position $\times$ group) were of interest. In case of the absence of significant three-way interactions or in case only two factors were defined in the ANOVA analysis, two-way interactions among the variables of interest (side $\times$ gender or position $\times$ gender) were explored. In the absence of any interaction effects, main effects (for side, gender or position) were analysed. Alpha was set on 0.05 for the ANOVA analysis and the independent tests. Post-hoc analyses were performed using a Bonferroni procedure when a significant difference was found with ANOVA.

All statistical analyses were performed using the statistical package for social sciences, version 16.0.

\section{RESULTS}

\section{Scapular inclination}

The results of the scapular upward rotation measurements are presented in table 1 . ANOVA for repeated measures did not show any significant three-way or two-way interaction effects. However, a significant side effect $(p<0.001)$ and position effect $(p<0.001)$ was found. There were no significant gender differences regarding this variable $(p=0.363)$. Across positions and for both genders, scapular upward rotation was larger on the dominant side compared with the non-dominant side. Post-hoc tests regarding the factor 'position' revealed that on both sides, and for both genders, scapular upward rotation significantly increased with increasing elevation of the arm $(p<0.001)$. 
REVAKI

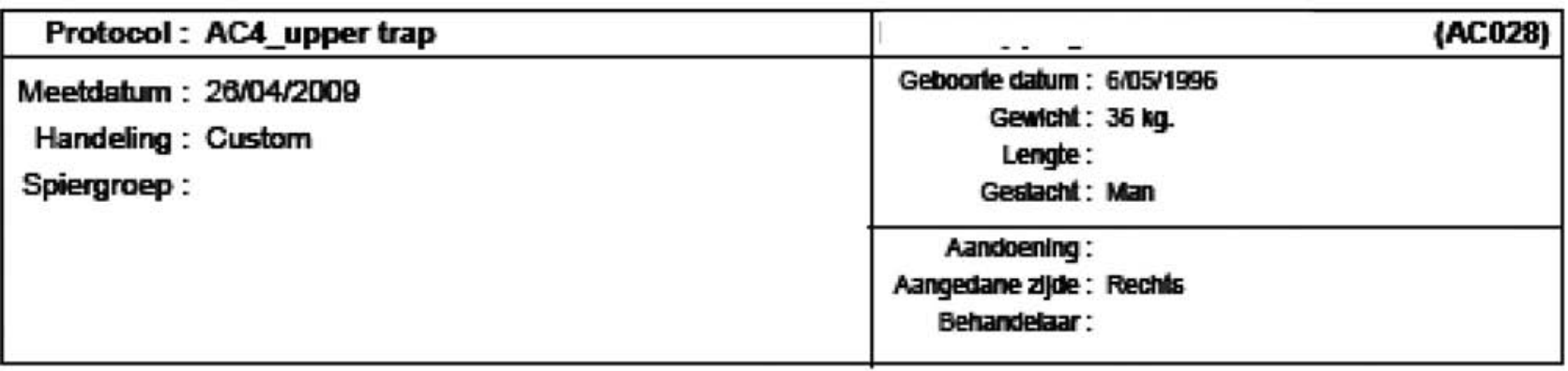

linker zijde

\begin{tabular}{|c|c|}
\hline Piekwaarde & \multicolumn{1}{c|}{ Tijd } \\
\hline $77,4 \mathrm{~N}$ & $3,80 \mathrm{sec}$ \\
$73,4 \mathrm{~N}$ & $1,53 \mathrm{sec}$ \\
\hline \multicolumn{2}{|c|}{ Gemiddeld : $75,4 \mathrm{~N}$} \\
to.v. gewicht : $2,1 \mathrm{~N} / \mathrm{kg}$ \\
\hline
\end{tabular}

rechter zijde

\begin{tabular}{|c|c|c|c|c|}
\hline \multicolumn{2}{|c|}{ Verschil } & \multirow[b]{2}{*}{+} & \multicolumn{2}{|l|}{ Piekwaarde } \\
\hline $24,9 \mathrm{~N}$ & $32,2 \%$ & & $102,3 \mathrm{~N}$ & $2,44 \mathrm{sec}$ \\
\hline \multirow[t]{2}{*}{$25.4 \mathrm{~N}$} & $34,5 \%$ & + & $98,8 \mathrm{~N}$ & $4,59 \mathrm{sec}$ \\
\hline & & & $\begin{array}{l}\text { Gem } \\
\text { t.o.v. ge }\end{array}$ & $\begin{array}{c}100,6 \mathrm{~N} \\
2,8 \mathrm{~N} / \mathrm{kg}\end{array}$ \\
\hline
\end{tabular}
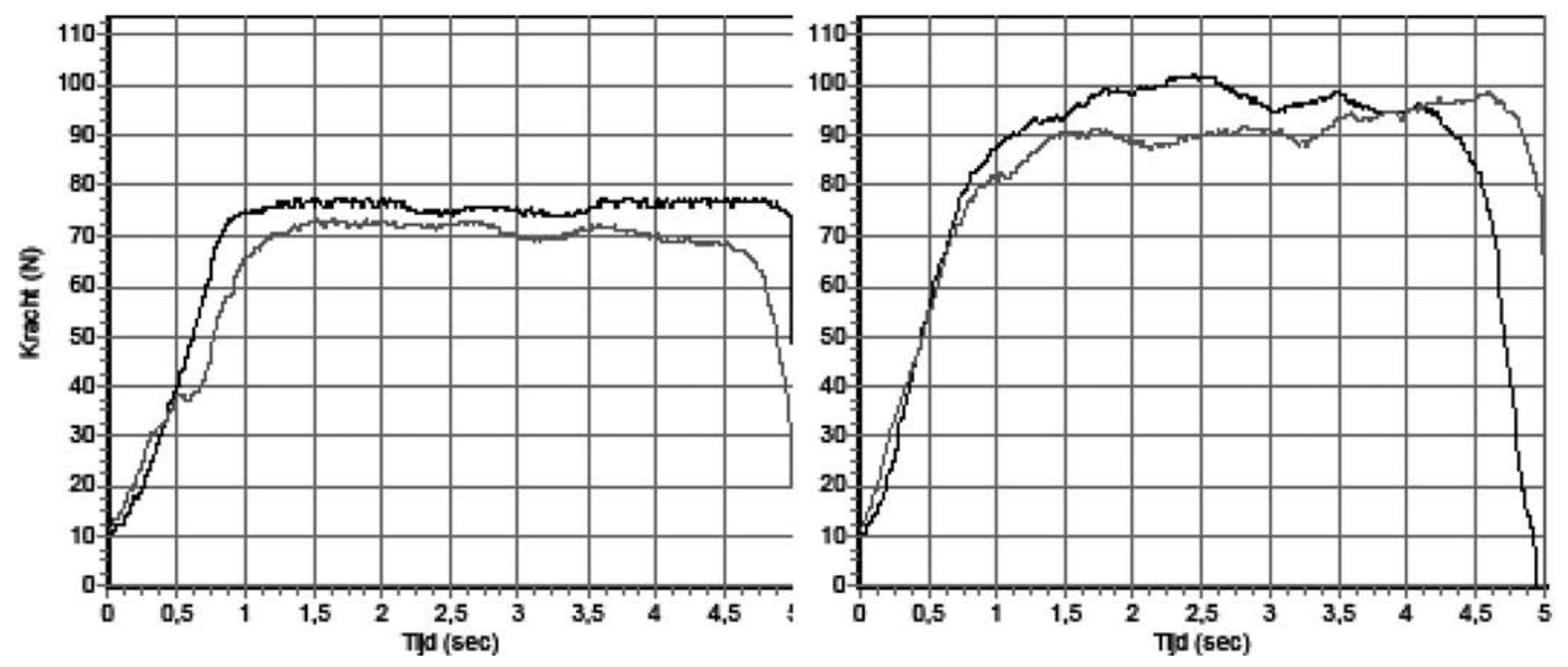

Figure 6 Example of a report concerning the manual muscle testing of the upper trapezius.

\section{Scapular muscle strength}

The descriptive analysis of the isometric muscle tests of the scapular muscles is presented in table 2 .

For UT muscle strength, ANOVA revealed no significant side $\times$ gender interaction effects $(p=0.957)$. Significant side effects were found ( $p=0.003)$, and for absolute muscle strength, also gender differences were significant $(p=0.018)$; however, for the normalised strength data, there were no significant gender differences $(p=0.117)$.

For MT and LT muscle strength, neither significant interaction effects, nor main side or gender differences were found, on the absolute as well as the normalised data.

For the SA muscle strength, the ANOVA for repeated measures also showed similar results for the absolute and the normalised data: no significant side $\times$ gender interaction effects, no significant gender differences; however, significant side differences ( $p=0.01$ and $p=0.009$ for the absolute and normalised data, respectively).

Data from the muscle balance ratios are presented in table 3 . Statistical analysis on the muscle balance ratios revealed no side $x$ gender interaction effects, nor main side or gender effects on any of the ratios calculated in this study.

\section{PM length}

The results from the PML measurements are summarised in table 4. Absolute muscle length (PML) data as well as PMI are noted. 


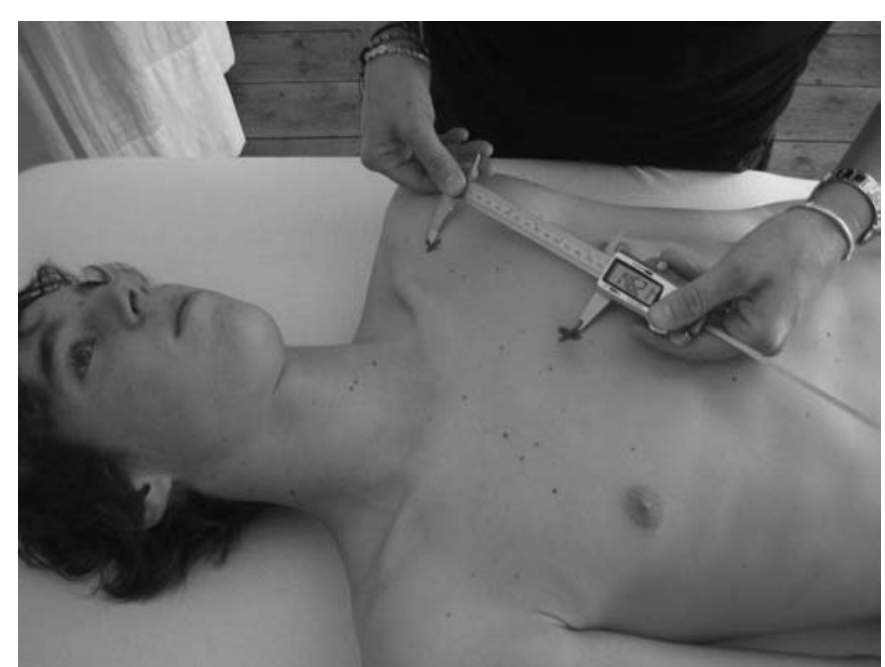

Figure 7 Measurement of pectoralis minor resting length. Written consent from the patient and his legal guardian was obtained for publication of the image in print and online.

Table 1 Means $( \pm S D$ ) for scapular upward rotation (in degrees) on the non-dominant and dominant side for male $(n=19)$ and female $(n=16)$ tennis players in three arm elevation positions in the scapular plane: $0^{\circ}$, $90^{\circ}$ and $180^{\circ}$

\begin{tabular}{|c|c|c|c|c|c|c|}
\hline & $0^{\circ}$ & & $90^{\circ}$ & & $180^{\circ}$ & \\
\hline & $\overline{N D}$ & D & $\overline{N D}$ & D & $\overline{N D}$ & D \\
\hline $\begin{array}{l}\text { Male } \\
\text { players }\end{array}$ & $3.5( \pm 2.6)$ & $5.2( \pm 4)$ & $24.2( \pm 6.2)$ & $29.2( \pm 10.4)$ & $53( \pm 7.8)$ & $54.1( \pm 9.1)$ \\
\hline $\begin{array}{l}\text { Female } \\
\text { players }\end{array}$ & $4.4( \pm 4)$ & $4.6( \pm 3)$ & $21.3( \pm 5.8)$ & $26.9( \pm 8.3)$ & $48.7( \pm 7.5)$ & $55.9( \pm 6.5)$ \\
\hline
\end{tabular}

D, dominant side; ND, non-dominant side.

Table 2 Means $( \pm S D)$ for absolute muscle strength $(\mathrm{N})$ and normalised muscle strength $(\mathrm{N} / \mathrm{kg})$ for UT, MT, LT and SA on the non-dominant and dominant side for male $(n=19)$ and female $(n=16)$ tennis players

\begin{tabular}{|c|c|c|c|c|}
\hline & \multicolumn{2}{|c|}{$\begin{array}{l}\text { Absolute muscle } \\
\text { strength (N) }\end{array}$} & \multicolumn{2}{|c|}{$\begin{array}{l}\text { Normalised muscle } \\
\text { strength }(\mathrm{N} / \mathrm{kg})\end{array}$} \\
\hline & ND & D & ND & D \\
\hline \multicolumn{5}{|l|}{ UT } \\
\hline $\begin{array}{l}\text { Male } \\
\text { players }\end{array}$ & $148.4( \pm 49.6)$ & $158.6( \pm 47.7)$ & $2.79( \pm 0.67)$ & $3( \pm 0.67)$ \\
\hline $\begin{array}{l}\text { Female } \\
\text { players }\end{array}$ & $112.9( \pm 33)$ & $123.5( \pm 36.7)$ & $2.46( \pm 0.55)$ & $2.68( \pm 0.64)$ \\
\hline \multicolumn{5}{|l|}{ MT } \\
\hline $\begin{array}{l}\text { Male } \\
\text { players }\end{array}$ & $36.6( \pm 10)$ & $35( \pm 11.3)$ & $0.71( \pm 0.16)$ & $0.68( \pm 0.14)$ \\
\hline $\begin{array}{l}\text { Female } \\
\text { players }\end{array}$ & $30.87( \pm 11.2)$ & $32.7( \pm 8.6)$ & $0.69( \pm 0.18)$ & $0.71( \pm 0.16)$ \\
\hline \multicolumn{5}{|l|}{ LT } \\
\hline $\begin{array}{l}\text { Male } \\
\text { players }\end{array}$ & $29.9( \pm 9.2)$ & $31.2( \pm 10.7)$ & $0.57( \pm 0.17)$ & $0.6( \pm 0.18)$ \\
\hline $\begin{array}{l}\text { Female } \\
\text { players }\end{array}$ & $27.9( \pm 10)$ & $27.9( \pm 9.2)$ & $0.61( \pm 0.21)$ & $0.61( \pm 0.21)$ \\
\hline \multicolumn{5}{|l|}{ SA } \\
\hline $\begin{array}{l}\text { Male } \\
\text { players }\end{array}$ & $136.8( \pm 44.8)$ & $154.8( \pm 61.9)$ & $2.66( \pm 0.8)$ & $2.94( \pm 1.02)$ \\
\hline $\begin{array}{l}\text { Female } \\
\text { players }\end{array}$ & $113.3( \pm 50.8)$ & $117.9( \pm 51.6)$ & $2.42( \pm 0.83)$ & $2.52( \pm 0.85)$ \\
\hline
\end{tabular}

D, dominant side; LT, lower trapezius; MT, middle trapezius; ND, non-dominant side; SA, serratus anterior; UT, upper trapezius.
No side $\times$ gender interaction effects $(p=0.398)$ were found; however, significant main gender $(p=0.006)$ and side $(p<0.001)$ differences were present regarding the absolute PML. Similar results were found regarding the PMI (interaction effect $p=0.631$, main effect gender $p=0.037$, main effect side $p<0.001$ ). All players have a significantly shorter PM on their dominant side, and across sides female players show shorter PML compared with male players.

\section{DISCUSSION}

The purpose of this investigation was to generate a descriptive profile of scapulothoracic strength, position and flexibility variables in a population of adolescent elite tennis players. Moreover, it was the purpose in this study to use field measurement tools to examine these variables, which were reproducible and easy to use in a clinical setting.

\section{Scapular upward rotation}

Our results show that young tennis players, both boys and girls have side differences regarding scapular upward rotation. In the three elevation positions, scapular upward rotation is higher on the dominant side compared with the non-dominant side. As it has been suggested that the presence of sufficient upward rotation during overhead movements is vital to injuryfree performance by clearing the acromion from the underlying subacromial structures, ${ }^{919} 20$ our results are in favour of optimal scapulohumeral kinematics, thus possibly protecting players from subacromial impingement. A possible explanation for the side differences could be found in the results of the muscle strength of the scapular rotators, in particular greater strength in the UT and SA. ${ }^{35}$ Our side differences confirm previously reported asymmetric resting scapular posture in healthy overhead athletes. ${ }^{19} 36$ Myers et al ${ }^{19}$ and Downar and Sauers ${ }^{36}$ also reported increased upward rotation on the dominant side in throwing athletes, and suggested that throwers develop chronic adaptation for more efficient performance of the throwing motion. In contrast, other researchers ${ }^{23} 37$ found a decrease in upward rotation in overhead athletes, suggesting that they are more prone to impingement-related shoulder pain.

In conclusion, a normal pattern of scapular upward rotation was found in young tennis players, with a side difference in favour of scapular inclination on the dominant side in all positions measured. However, we did not measure other elevation angles with respect to scapular inclination.

\section{Scapular muscle strength}

The major result of our strength measurement is the fact that in these young elite tennis players significant side differences were found for UT and SA strength, whereas MT and LT did not show significant side differences. Only a few authors have examined scapular muscle strength in swimmers, ${ }^{24}$ baseball pitchers $^{38}$ and gymnasts, ${ }^{39}$ with conflicting results. On the basis of our results, we might suggest to coaches and clinicians to emphasise symmetric strength training of the scapular stabilisers, in particular MT and LT, to maintain the balance between the dominant and non-dominant side with respect to stabilising and moving muscles in these players. Stronger UT and SA on the dominant side might possibly lead to muscle imbalances, increasing the risk of injury.

Besides reporting the absolute and normalised strength data, we calculated muscle balance ratios in the scapular muscles. As this is the first study to add balance ratios to the data 
Table 3 Means ( \pm SD) for the muscle balance ratios UT/MT, UT/LT, UT/SA and LT/SA on the non-dominant and dominant side for male ( $n=19$ ) and female $(n=16)$ tennis players

\begin{tabular}{|c|c|c|c|c|c|c|c|c|}
\hline & \multicolumn{2}{|l|}{ UT/MT } & \multicolumn{2}{|l|}{ UT/LT } & \multicolumn{2}{|l|}{ UT/SA } & \multicolumn{2}{|l|}{ LT/SA } \\
\hline & $\overline{\text { ND }}$ & D & ND & D & ND & D & ND & D \\
\hline Male players & $4.14( \pm 1.18)$ & $4.79( \pm 1.71)$ & $5.29( \pm 2.19)$ & $5.62( \pm 2.66)$ & $1.12( \pm 0.32)$ & $1.11( \pm 0.39)$ & $0.21( \pm 0.09)$ & $0.20( \pm 0.1)$ \\
\hline Female players & $4.47( \pm 3.38)$ & $3.97( \pm 1.03)$ & $4.32( \pm 1.25)$ & $4.73( \pm 1.25)$ & $1.08( \pm 0.35)$ & $1.13( \pm 0.35)$ & $0.26( \pm 0.15)$ & $0.24( \pm 0.12)$ \\
\hline
\end{tabular}

D, dominant side; LT, lower trapezius; MT, middle trapezius; ND, non-dominant side; SA, serratus anterior; UT, upper trapezius.

Table 4 Means $( \pm S D)$ for PML $(\mathrm{cm})$ and the PMI $(\mathrm{PML} /$ body length $\times$ $100)$ on the non-dominant and dominant side for male $(n=19)$ and female $(n=16)$ tennis players

\begin{tabular}{llllll}
\hline & PML $(\mathbf{c m})$ & & & PMI & \\
\cline { 2 - 3 } \cline { 5 - 6 } & ND & D & & ND & D \\
\hline Male players & $12.9( \pm 1.3)$ & $11.7( \pm 1.2)$ & & $7.9( \pm 0.4)$ & $7.1( \pm 0.4)$ \\
Female players & $11.7( \pm 0.89)$ & $10.8( \pm 1.2)$ & & $7.5( \pm 0.4)$ & $6.9( \pm 0.7)$ \\
\hline
\end{tabular}

D, dominant side; ND, non-dominant side; PMI, pectoralis minor index; PML, pectoralis minor muscle length.

on isometric measurements, using the described protocol, we have no literature to compare our results with. The interpretation of the balance ratios, as discussed in this study, should be limited to side comparisons within one player, or comparing individual player's balance ratios with these data, on the condition that identical procedures of testing have been followed.

\section{PM length}

Our results show significantly shorter PML on the dominant side compared with the non-dominant side in both female and male players. In addition, the female players have lower values compared with the male players. Researchers have speculated that the PM, through increased active or passive tension, may affect normal scapular kinematics. ${ }^{40-42}$ Borstad and Ludewig ${ }^{34}$ demonstrated that the scapular motion alterations in subjects with a relatively short PM are consistent with those previously demonstrated by subjects with impingement.

The side differences found in our players might be the result of adaptive changes in the muscle tension of the PM due to repetitive powerful use of that muscle performing protraction during the tennis serve and strike.

In view of our results, we might encourage coaches and physiotherapists to include PM stretches ${ }^{4344}$ in the preventive stretching programme, starting at a young age.

However, it must be noted that, in spite of statistical significance, side differences are $0.9 \mathrm{~cm}$ for the female players and $1.2 \mathrm{~cm}$ for the male players. One might question the clinical relevance of this result. Statistical difference was reached because side differences, although small, were present in all but one player. In view of this assumption, our results should be interpreted with caution, and future studies should examine PM tightness more thoroughly in view of clinical relevance.

\section{Limitations of the study and future investigations}

We used measurement techniques and protocols, which are field measurement tools, easy to transport and to use in a sports setting on a tennis court or training area, and affordable for clinicians, who often do not have access to the high-technology equipment used in research centres and academic university settings. This is one of the strengths of the study, giving the

\section{What is already known on this topic}

Scapular dyskinesis in tennis players possibly increases the risk of shoulder pathology. Therefore it is important to evaluate scapular position and motion variables in healthy tennis players.

\section{What this study adds}

Elite adolescent tennis players have increased scapular upward rotation on their dominant side, side differences in UT and SA strength, but symmetrical MT and LT strength and relatively short PM on the dominant side. These results may assist the clinician in the musculoskeletal screening of young tennis players.

possibility to clinicians to reproduce the tests in practice, and use our results as a reference base in the evaluation of young tennis players, but this also leads to a limitation. Clinical measures often do not achieve the same reliability, validity and accuracy compared with laboratory investigations; therefore we encourage clinicians to provide maximal standardisation and reliability in reproducing the tests in this study by limiting the testing to one examiner, blinding the results during testing, performing pilot reliability studies if necessary and maximising standardisation in palpation, subject position and task instruction during testing.

A second limitation is the lack of a control group. A number of the variables examined in this study were explored for the first time. Therefore, future studies should compare the results found in this investigation with age-matched non-athletic young subjects and recreational tennis players. In addition, a longitudinal follow-up of the measurements performed is mandatory, and further detailed results based on specific stroke techniques, for instance one versus two-handed backhand strokes. Yearly investigation of these variables in elite tennis players, as well as investigation of the same level of players but an age category higher (15-18 years) may add valuable information about scapular behaviour in elite tennis players. Moreover, additional testing would give the opportunity over time to provide some normative data for future clinical screening of tennis players.

Acknowledgements The authors wish to thank Mikael Stripple, Julius Demburg, Johan Hedsberg and Johan Kareld, coaches from the Swedish Tennis Federation, for their collaboration in the acquisition of the data, and Båstad Sportcenter for the use of their facilities during the investigation. 


\section{Competing interests None.}

Ethics approval This study was conducted with the approval of Ghent University Ethics Committee.

Provenance and peer review Not commissioned; externally peer reviewed.

Patient consent Obtained.

\section{REFERENCES}

1. Kibler WB, Safran MR. Musculoskeletal injuries in the young tennis player. Clin Sports Med 2000;19:781-92.

2. Pluim BM, Staal JB, Windler GE, et al. Tennis injuries: occurrence, aetiology, and prevention. Br J Sports Med 2006;40:415-23.

3. Baxter-Jones A, Maffulli N, Helms P. Low injury rates in elite athletes. Arch Dis Child 1993;68:130-2.

4. Sciascia A, Kibler WB. The pediatric overhead athlete: what is the real problem? Clin J Sport Med 2006;16:471-7.

5. Borsa PA, Laudner KG, Sauers EL. Mobility and stability adaptations in the shoulder of the overhead athlete: a theoretical and evidence-based perspective. Sports Med 2008;38:17-36.

6. Cools AM, Declercq G, Cagnie B, et al. Internal impingement in the tennis player: rehabilitation guidelines. Br J Sports Med 2008;42:165-71.

7. Kibler WB, Press J, Sciascia A. The role of core stability in athletic function. Sports Med 2006;36:189-98.

8. Lintner D, Noonan TJ, Kibler WB. Injury patterns and biomechanics of the athlete's shoulder. Clin Sports Med 2008;27:527-51.

9. Kibler WB. The role of the scapula in athletic shoulder function. Am J Sports Med 1998;26:325-37.

10. Kibler WB, McMullen J. Scapular dyskinesis and its relation to shoulder pain. J Am Acad Orthop Surg 2003;11:142-51.

11. Ellenbecker TS, Roetert EP, Piorkowski PA, et al. Glenohumeral joint internal and external rotation range of motion in elite junior tennis players. J Orthop Sports Phys Ther 1996;24:336-41.

12. Ellenbecker TS, Roetert EP, Bailie DS, et al. Glenohumeral joint total rotation range of motion in elite tennis players and baseball pitchers. Med Sci Sports Exerc 2002:34:2052-6.

13. Kibler WB, Chandler TJ, Livingston BP, et al. Shoulder range of motion in elite tennis players. Effect of age and years of tournament play. Am J Sports Med 1996:24:279-85.

14. Torres RR, Gomes JL. Measurement of glenohumeral internal rotation in asymptomatic tennis players and swimmers. Am J Sports Med 2009;37:1017-23.

15. Vad VB, Gebeh A, Dines D, et al. Hip and shoulder internal rotation range of motion deficits in professional tennis players. J Sci Med Sport 2003;6:71-5.

16. Codine P, Bernard PL, Pocholle M, et al. Influence of sports discipline on shoulder rotator cuff balance. Med Sci Sports Exerc 1997;29:1400-5.

17. Ellenbecker T, Roetert EP. Age specific isokinetic glenohumeral internal and external rotation strength in elite junior tennis players. J Sci Med Sport 2003;6:63-70.

18. Silva RT, Gracitelli GC, Saccol MF, et al. Shoulder strength profile in elite junior tennis players: horizontal adduction and abduction isokinetic evaluation. $\mathrm{Br} \mathrm{J}$ Sports Med 2006; 40:513-17; discussion 517.

19. Myers JB, Laudner KG, Pasquale MR, et al. Scapular position and orientation in throwing athletes. Am J Sports Med 2005;33:263-71.

20. Silva RT, Hartmann LG, Laurino CF et al. Clinical and ultrasonographic correlation between scapular dyskinesia and subacromial space measurement among junior elite tennis players. Br J Sports Med. Published Online First: 8 April 2008. doi:10.1136/bjsm.2008.046284.
21. Johnson MP, McClure PW, Karduna AR. New method to assess scapular upward rotation in subjects with shoulder pathology. J Orthop Sports Phys Ther 2001;31:81-9.

22. Borsa PA, Timmons MK, Sauers EL. Scapular-positioning patterns during humeral elevation in unimpaired shoulders. J Ath/ Train 2003;38:12-17.

23. Laudner KG, Stanek JM, Meister K. Differences in scapular upward rotation between baseball pitchers and position players. Am J Sports Med 2007;35:2091-5

24. Su KP, Johnson MP, Gracely EJ, et al. Scapular rotation in swimmers with and without impingement syndrome: practice effects. Med Sci Sports Exerc 2004;36:1117-23.

25. Thomas SJ, Swanik KA, Swanik C, et al. Glenohumeral rotation and scapular position adaptations after a single high school female sports season. J Athl Train 2009;44:230-7.

26. Sullivan SJ, Chesley A, Hebert G, et al. The validity and reliability of hand-held dynamometry in assessing isometric external rotator performance. J Orthop Sports Phys Ther 1988;10:213-17.

27. Tyler TF, Nahow RC, Nicholas SJ, et al. Quantifying shoulder rotation weakness in patients with shoulder impingement. J Shoulder Elbow Surg 2005;14:570-4.

28. Michener LA, Boardman ND, Pidcoe PE, et al. Scapular muscle tests in subjects with shoulder pain and functional loss: reliability and construct validity. Phys Ther 2005;85:1128-38.

29. Hislop HJ, Montgomery J, Connelly B. Daniels and Worthingham's muscle testing techniques of manual examination, 6th edn. Philadelphia, Pennsylvania, USA: WB Saunders Co, 1995.

30. Donatelli R, Ellenbecker TS, Ekedahl SR, et al. Assessment of shoulder strength in professional baseball pitchers. J Orthop Sports Phys Ther 2000;30:544-51.

31. Kendall FP, McCrerary EK, Provance PG. Muscles, testing and function: with posture and pain. Baltimore, Maryland, USA: Williams \& Wilkins, 1993.

32. Mullaney MJ, McHugh MP, Donofrio TM, et al. Upper and lower extremity muscle fatigue after a baseball pitching performance. Am J Sports Med 2005;33:108-13.

33. Borstad JD. Measurement of pectoralis minor muscle length: validation and clinical application. J Orthop Sports Phys Ther 2008;38:169-74.

34. Borstad JD, Ludewig PM. The effect of long versus short pectoralis minor resting length on scapular kinematics in healthy individuals. J Orthop Sports Phys Ther 2005:35:227-38.

35. Johnson G. Anatomy and actions of the trapezius muscle. Clin Biomech 1994:9:44-50.

36. Downar JM, Sauers EL. Clinical measures of shoulder mobility in the professional baseball player. J Athl Train 2005;40:23-9.

37. Oyama $\mathbf{S}$, Myers JB, Wassinger CA, et al. Asymmetric resting scapular posture in healthy overhead athletes. J Athl Train 2008;43:565-70.

38. Trakis JE, McHugh MP, Caracciolo PA, et al. Muscle strength and range of motion in adolescent pitchers with throwing-related pain: implications for injury prevention. Am J Sports Med 2008;36:2173-8.

39. Cools AM, Geerooms E, Van den Berghe DF, et al. Isokinetic scapular muscle performance in young elite gymnasts. J Athl Train 2007;42:458-63.

40. Hébert LJ, Moffet H, McFadyen BJ, et al. Scapular behavior in shoulder impingement syndrome. Arch Phys Med Rehabil 2002;83:60-9.

41. Borstad JD. Resting position variables at the shoulder: evidence to support a posture-impairment association. Phys Ther 2006;86:549-57.

42. Ludewig PM, Cook TM, Nawoczenski DA. Three-dimensional scapula orientation and muscle activity at selected positions of humeral elevation. J Orthop Sports Phys Ther 1996;24:57-65.

43. Ellenbecker TS, Cools AM. Rehabilitation of shoulder impingement syndrome and rotator cuff injuries: an evidence based review. Br J Sports Med 2010;44:319-27.

44. Borstad JD, Ludewig PM. Comparison of three stretches for the pectoralis minor muscle. J Shoulder Elbow Surg 2006;15:324-30. 